\title{
Aux frontières de la parenté
}

\section{Une réflexion sur l'usage des marges dans les études contemporaines sur la parenté}

\author{
Anaïs Martin, Léa Linconstant, Anne-Sophie Giraud, Hélène \\ Malmanche, Manon Vialle
}

Émulations - Revue de sciences sociales

2019, n 32, «Aux frontières de la parenté. Un éclairage par les marges ».

\section{Article disponible à l'adresse suivante}

https://ojs.uclouvain.be/index.php/emulations/article/view/martinetal

\section{Pour citer cet article}

Anaïs Martin, Léa Linconstant, Anne-Sophie Giraud, Hélène Malmanche, Manon Vialle, «Aux frontières de la parenté. Une réflexion sur l'usage des marges dans les études contemporaines sur la parenté », Émulations, n 32, Mise en ligne le 20 avril 2020. DOI : 10.14428/emulations.032.01

Distribution électronique : Université catholique de Louvain (Belgique) : ojs.uclouvain.be

(c) Cet article est mis à disposition selon les termes de la Licence Creative Commons Attribution, Pas d'Utilisation Commerciale 4.0 International. http://creativecommons.org/licenses/by-nc/4.0/

Éditeur : Émulations - Revue de sciences sociales / Presses universitaires de Louvain https://ojs.uclouvain.be/index.php/emulations

ISSN électronique : $1784-5734$

PUL PRESSES

UNIVERSITAIRES

DE LOUVAIN 


\title{
Aux frontières de la parenté Une réflexion sur l'usage des marges dans les études contemporaines sur la parenté
}

\author{
Anaïs Martin ${ }^{1}$, Léa Linconstant ${ }^{2}$, \\ Anne-Sophie Giraud ${ }^{3}$, Hélène Malmanche ${ }^{4}$, \\ Manon Vialle ${ }^{5}$
}

\begin{abstract}
[Résumé] Que peut nous apprendre la question des marges sur la parenté ? Réciproquement, comment les études sur la parenté contribuent-elles à éclairer le rapport des marges à la norme ? Comment cette notion de marge, ou encore celles de confins, de frontières et de limites sont-elles comprises selon les époques et les espaces géographiques ? À travers cinq articles originaux et un double entretien, ce numéro consacré aux « frontières de la parenté » vise à élargir les bases d'une réflexion amorcée dès les débuts de l'anthropologie. II éclaire la diversité des acceptions de la notion de marge par la description des pratiques, des représentations et des normes qui leurs sont associées. La marge apparaît d'abord comme un objet à ethnographier et/ou comme un outil analytique. Elle est ensuite questionnée en tant que catégorie d'action. Enfin, la dimension processuelle et temporelle des statuts et des situations marginales est examinée.
\end{abstract}

Mots-clés : marges, parenté, filiation, mariage, mort.

At the edges of kinship:

A reflection on the use of margins in contemporary kinship studies

[Abstract] What can we learn about kinship from the question of margins? Conversely, how do kinship studies help to inform the relation of margins to the norm? How are understood this notion of margin, or those of edges, boundaries, and limits, across time and geographical spaces? Through five original articles and a double interview, this issue devoted to the "frontiers of kinship" aims to broaden a reflection that has been a point of discussion since the beginning of social anthropology. It sheds light on the diversity of meanings of the notion of margin by describing the practices, representations and standards associated with them. The margin appears first as an object to be observed and described and/or as an analytical tool. It is then questioned as a category of action. Finally, the procedural and temporal dimension of statuses and marginal situations is examined.

Keywords: margins, kinship, descent, marriage, death.

Que peut nous apprendre la question des marges sur la parenté ? Réciproquement, comment les études sur la parenté contribuent-elles à éclairer le rapport des marges à la norme ? Comment cette notion de marge, ou encore celles de confins, de frontières,

\footnotetext{
${ }^{1}$ École des hautes études en sciences sociales, Centre Norbert Elias, France.

${ }^{2}$ Aix-Marseille Université, IDEMEC, France.

${ }^{3}$ Centre national de la recherche scientifique, LISST-CAS, France.

${ }^{4}$ École des hautes études en sciences sociales, CESPRA, France.

${ }^{5}$ Centre national de la recherche scientifique, Centre Norbert Elias, France.
} 
et de limites sont-elles comprises à travers les époques et les espaces géographiques ? Ces questionnements en miroir ont émergé des discussions qui se tiennent depuis novembre $2017 \mathrm{au}$ sein du groupe de recherche EnCoRe (Engendrement, Corps, Relations) $)^{6}$. Réunies autour de l'étude des techniques de procréation assistée (TPA) et de la parenté euro-américaine ${ }^{7}$ contemporaine, nous avons souhaité établir dans ce numéro porté par les cinq membres d'EnCoRe, un dialogue entre différentes disciplines, champs de recherche et aires culturelles. Cette approche résolument comparative et interdisciplinaire était au cœur du travail de notre défunt collègue et professeur Enric Porqueres i Gené, anthropologue de la parenté et de la personne dont les recherches ont considérablement enrichi le champ francophone et bien au-delà. Nous dédions ce numéro à sa mémoire et la parole est donnée à son ancienne étudiante Noémie Merleau-Ponty, aujourd'hui chargée de recherche au CNRS, pour lui rendre un hommage personnel.

À travers cinq articles originaux et un double entretien, ce numéro thématique consacré aux « frontières de la parenté » vise à élargir les bases d'une réflexion amorcée dès les débuts de l'anthropologie. L’organisation des sociétés et de leurs limites internes (prescriptions et prohibitions matrimoniales, division du travail, organisation rituelle, etc.) est au cœur des préoccupations des ethnologues depuis la naissance de la discipline, dans laquelle la parenté occupe une place majeure (à titre d'exemple : LéviStrauss, 1967 ; Malinowski, 1963 ; Morgan, 1985). À cet égard, les marges ont fait l'objet d'une attention particulière notamment lorsqu'elles sont en rapport avec le cycle de vie : passage à l'âge adulte, enfantement et décès sont autant d'étapes scandées par des rites et pratiques de parenté donnant à voir des situations marginales ou liminaires (Gennep, 1991). Arnold van Gennep, qui est l'un des premier·e·s à s'être intéressé de près à ces rites de passage, définit la liminarité comme la période intermédiaire entre deux statuts (passage de la vie in utero à la vie extra-utérine, de l'adolescence à l'âge adulte, etc.). Phase transitoire toujours dangereuse, la marge est un entre-deux à la fois temporel, spatial et symbolique. Malgré les critiques adressées à son analyse (Gluckman, 1962 ; La Fontaine, 1977), les idées d’A. van Gennep ont connu d'importants développements et ont notamment nourri la réflexion de Victor Turner (1990). Ce dernier s'est attaché à décrire la liminarité dans le cadre des rituels d'initiation des jeunes hommes : ambiguïté sexuelle, humilité, abolition de la hiérarchie ou encore vie en communitas ${ }^{8}$ caractérisent cette phase d'entre-deux et contrastent fortement avec l'organisation sociale qui prévaut en dehors de ces périodes liminaires. Par ailleurs, dans une approche centrée sur les institutions et l'action rituelle, Mary Douglas (1966) a exploré le rap-

\footnotetext{
${ }^{6}$ Groupe d'étude autour de l'approche relationnelle du corps et de l'engendrement. Voir EnCoRe. En ligne, https://encore.hypotheses.org.

${ }^{7}$ Pour une discussion sur la notion de parenté « euro-américaine » voir Edwards, 2006.

8 Pour V. Turner (1990), la liminarité se caractérise par l'absence de structure et de hiérarchie sociales : pendant des périodes spécifiques plus ou moins longues (un rituel par exemple), des individus désignés se retrouvent aux marges de leur société dans le sens où ils ne sont plus soumis aux règles sociales de leur groupe. La communitas constitue le mode relationnel particulier aux situations liminaires, dans lequel tous les individus sont égaux et les statuts indifférenciés.
} 
port entre marges (ou confins), ordre social et normes, à partir d'une réflexion sur les processus classificatoires des sociétés. S’intéressant plus particulièrement à la notion de pollution et de tabou, elle dessine les contours d'une zone de confins dans laquelle est repoussé ce qui est considéré comme " dangereux », "sale » ou encore " profane ». Espace d'incertitude situé à la fois aux frontières extérieures et intérieures de l'ordre social, les confins forment un continuum ni tout à fait en dedans ni tout à fait en dehors de la société (Douglas, 1966) : les marges semblent ainsi consubstantielles à l'existence de la vie sociale.

Les anthropologues ont souvent utilisé les marges comme terrain d'observation des personnes, des relations, des corps sociaux et plus largement de la vie elle-même (Kaufman, Morgan, 2005). L'étude de situations « en marge » a d'ailleurs contribué au renouvellement des recherches en anthropologie de la parenté, qui avaient été fortement mises à mal par la première critique de David Schneider (1984). Mettant en lumière le biais ethnocentrique avec lequel les systèmes de parenté des sociétés dites holistes (ou traditionnelles) avaient été étudiés jusqu'alors, D. Schneider a montré comment des catégories et prénotions euro-américaines avaient été appliquées à l'analyse sans que les conséquences pour les descriptions anthropologiques n'aient été aperçues (Wilgaux, 2005). Avec l'étude de la personne et la question du genre (Carsten, 2004), les TPA ont participé à l'émergence des new kinship studies par l'analyse de nouvelles configurations, qui pourraient être qualifiées de « marginales», telles que les familles adoptives (à titre d'exemple : Carsten, 2000 ; Fine, 1998), les familles recomposées (à titre d'exemple : Cherlin, 1978 ; Martial, 2003), les familles ayant eu recours à une procréation avec tiers donneur (à titre d'exemple : Martin, 2019 ; Mathieu, 2017 ; Théry 2010) ou à une gestation pour autrui (à titre d'exemple : Courduriès, 2016; Lavoie, 2019), ou encore les parentés trans (Hérault, 2011). Traduisant des espaces d'incertitude ou de transition dans leur dimension temporelle, symbolique et/ou normative, ces situations « aux confins » de la parenté mettent en lumière les changements contemporains dans les représentations et normes sociales relatives à la famille et à l'engendrement (Ginsburg, Rapp, 1995 ; Fine, 2002 ; Franklin, 1997 ; Strathern, 1992). Le modèle de parenté euro-américain supposément « naturel », reposant sur l'analogie entre la reproduction sexuée de l'espèce humaine et le lien parent-enfant qui en découlerait, est mis en question par des situations où procréation et parenté sont dissociées : dans l'adoption, les recompositions familiales ou encore l'engendrement avec tiers donneur, des personnes participent à la procréation sans devenir parents et inversement. La redéfinition des statuts de père et de mère contribue à la redéfinition même des catégories d'hommes et de femmes et de la distinction féminin/masculin. En favorisant l'émergence d'une pluralité de statuts de parenté et de modes d'établissement de la filiation, ces configurations familiales « hors-normes » produisent de nouvelles manières d'envisager le genre et la parenté (Edwards et al., 1999).

Consacré à la recherche francophone, ce numéro se veut didactique. Il propose de cheminer parmi des enquêtes empiriques aux thématiques et approches variées 
(histoire, travail social, sociologie, anthropologie) qui ouvrent la voie à des questions conceptuelles et méthodologiques. L’entretien accordé par Laurence Hérault (anthropologue) et Irène Théry (sociologue) en est un exemple marquant. Les travaux présentés dans les articles de Marie-Lise Fieyre (historienne), Delphine Manetta (anthropologue), Lucie Jégat (sociologue), Anne Lavanchy (anthropologue), ainsi qu'Isabel Côté (travailleuse sociale), Martine Gross (sociologue), Kévin Lavoie (travailleur social) et Line Chamberland (sociologue) éclairent la diversité des acceptions de la notion de marge par la description des pratiques, des représentations et des normes qui leurs sont associées. Trois axes se dégagent des pistes de réflexion ouvertes par ces articles ; la marge est d'abord appréhendée comme objet à ethnographier et/ou comme outil analytique (1) ; elle est ensuite questionnée en tant que catégorie d'action (2) ; enfin, l'accent est mis sur la dimension processuelle et temporelle des statuts et des situations en marge (3).

\section{Les marges entre objet ethnographique et outil analytique}

Les articles constituant ce numéro visent à appréhender des situations marginales et à les documenter en tant que telles, qu'il s'agisse de la constitution de mariages " mixtes », de la perte d'un·e enfant, ou encore des enfants illégitimes chez les Bourbon à la fin du Moyen Âge. L'article d'A. Lavanchy permet de documenter et d'analyser la façon dont l'administration suisse perçoit et traite les mariages de couples « mixtes », unissant une personne suisse à une personne de nationalité étrangère. L'étude de ces mariages "qui dérangent » (Porqueres i Gené, 2001) renseigne sur les stéréotypes, à la fois culturels et genrés, qui accompagnent la conjugalité mixte. Ces unions renseignent ainsi sur la façon dont les institutions se représentent la nation. L. Jégat, quant à elle, donne à voir le vécu des parents en deuil d'enfant, dans un contexte français où la ritualité contemporaine minimale autour de la mort rendrait difficile la sortie du deuil, plus encore lorsqu'il s'agit de morts exceptionnelles. En étudiant cette " parentalité liminale » (dans ce numéro : 51), elle éclaire les frontières de la famille et interroge les rôles et les obligations genrées propres au statut de parent. M.-L. Fieyre, de son côté, montre que les bâtards nobles chez les Bourbon à la fin du Moyen Âge sont « mis à la marge sans être marginalisés » (dans ce numéro : 20), repoussés aux frontières de la parenté. Ils se situent « in margine : sur (ou à) la marge »(dans ce numéro : 22). Si les bâtard·e.s sont maintenu.e.s dans une position d'infériorité par rapport aux enfants légitimes, ils ne sont toutefois pas exclus de l'ensemble des successions. Un·e bâtard·e pourra, par exemple, hériter des armoiries d'un ancêtre et ainsi de son capital symbolique sans pour autant hériter de ses biens. L'autrice montre que la frontière n'est pas une simple séparation entre parenté légitime et illégitime : mobile, variable et ajustable, elle peut contribuer, comme dans le cas étudié, à une redéfinition et une multiplication des frontières de la parenté. Ces articles permettent donc d'interroger la notion même de marge : s'apparente-t-elle à un objet qui se situerait en périphérie de la société ? Comment contribue-t-elle à éclairer la façon dont les normes se construisent? 
L'étude des situations marginales permet en outre de mettre en lumière la norme dominante, comme le soulignent I. Théry et L. Hérault dans l'entretien qu'elles ont accordé au groupe EnCoRe. On ne peut étudier les marges sans comprendre la manière dont leur marginalité se construit par rapport à la norme : le statut de beau-parent éclaire celui de parent, comme la parenté trans met en lumière la parenté cis. Dans toutes les sociétés, les marges mettent en lumière les complexités et les contradictions d'un système ainsi que les idéologies normatives de la notion de personne, de parent, ou encore d'homme et de femme (Douglas, 1966 ; Conklin, Morgan, 1996). Leur analyse rend explicites des normes qui n'étaient pas jusqu'alors interrogées et permet ainsi d'appréhender la construction de leurs frontières et d'éclairer leurs plasticités.

\section{Marges et catégories d'action}

Être en ou à la marge produit des effets sur les statuts, les relations et les actions des personnes concernées et, au-delà, sur l'ensemble du groupe familial et social. Cette position particulière peut empêcher d'agir ou, au contraire, permettre une plus grande capacité d'action. V. Turner présentait ainsi la liminalité comme un espace de "puissance et de potentialité », « d'expérimentation et de jeu » pouvant de ce fait être instable et dangereuse (1990: 33-34).

Dans le cas des bâtard·e.s de Bourbons, des familles lesboparentales et de leurs donneurs en France, ou encore des groupes de parent·e-s jaàna au Burkina Faso, la marginalité peut permettre aux acteurs et actrices en présence une plus grande latitude ou une plus grande flexibilité d'action. Chez les Bourbon, les bâtard·e.s possédaient certains avantages politiques et participaient au maintien de la société. C’est notamment le cas lorsqu'ils occupaient des rôles que ne peuvent tenir les enfants légitimes ou encore lorsqu'ils contractaient des unions matrimoniales dans le but de maintenir et renforcer les liens existants entre le chef de lignage et ses vassaux. Les avantages politiques des enfants illégitimes constituaient par conséquent un atout indispensable pour celles et ceux qui sont légitimes. Leur statut leur conférait une plus grande liberté d'action. On retrouve dans la période contemporaine et sous une autre forme cette liberté que produit la marge. Dans le cas des familles lesboparentales en France étudiées par I. Côté et al., la pluralité des liens d'apparentement qui les caractérisent témoigne de la plasticité des frontières de la parenté. L'article nous renseigne sur les configurations des familles qui ont eu recours à un donneur de sperme en France, dans un contexte où seul l'anonymat du donneur est institutionnalisé'. En raison de l'interdiction de prise en charge des familles lesboparentales dans les services d'infertilité et de la difficulté rencontrée par la société française à penser la diversité des positions que peut endosser le donneur, les personnes font preuve de créativité, afin de trouver une place particulière pour ce donneur dont le statut n’est pas encore pleinement institué. Cette

${ }^{9}$ Le projet de révision des lois relatives à la bioéthique, actuellement en discussion au Parlement français, prévoit l'ouverture d'un accès aux origines pour les personnes conçues par don nées après la révision. 
marginalité est ici un moteur pour agir : dans un contexte d'« institution incomplète » (Cherlin, 1978), la place du donneur est à inventer et à ajuster par rapport aux modèles existants. Tout comme il semble impossible de comprendre la place du donneur sans comprendre ce qui fonde la parenté et la parentalité, il est impossible de comprendre la mobilité des personnes sans prendre en compte le fondement de la parenté jaàna au Burkina Faso. D. Manetta constate que les (im)mobilités des personnes dépendent de catégories de genre et d'âge différentes et qu'elles participent à la définition des frontières de la parenté. Dans un système de résidence virilocale, la mobilité des femmes est l'objet de négociations entre groupes de parenté, d'autant plus lorsque le mariage n'est pas celui initialement prévu par la famille. L'autrice montre que les transformations des frontières de la parenté sont le fruit du mouvement des personnes, au fil du temps, dans un ensemble de lieux pluriels et morcelés.

\section{Marges et temporalité}

Les notions de marge, de liminalité et de frontières mettent l'accent sur les dimensions processuelles et temporelles des statuts, et paradoxalement sur l'atemporalité qui semble toucher certaines des situations en marge. Que cela soit dans le cadre des parents en deuil d'enfant, des couples mariés mixtes ou des donneurs en France, le statut des personnes en marge semble entaché par cette marginalité, de manière durable. En raison du statut impensé des parents qui perdent un.e enfant, ces personnes sont maintenues de façon indéterminée dans un statut liminal, comme le montre L. Jégat. Le deuil qui les touche ne trouve pas de résolution, il devient atemporel et leur fait acquérir, pour un temps indéterminé, un statut qui reste normalement momentané. Ces situations « hors-normes » s'inscrivent donc dans le temps et peuvent être mouvantes. Pour l'illustrer, L. Jégat préfère la notion de liminalité de V. Turner (1990) à celle de marge. La notion de liminalité, explique-t-elle, « permet d'explorer des processus, des espaces de transitions entre les normes sociales et les normes institutionnelles » (dans ce numéro : 51). L'autrice souligne cette dimension processuelle et temporelle à travers l'usage des termes de « deuilleurs » et « deuilleuses ». Elle montre que la perte de l'enfant n'empêche pas ces dernier.e-s d'agir selon un registre d'actions propre à la parentalité, et de continuer certaines des activités de soin liées à leur statut de parents. Dans l'article d'A. Lavanchy, les personnes naturalisées suite à des mariages mixtes en Suisse semblent être elles aussi enferrées dans une position marginale. Le mariage donne la possibilité au conjoint ou à la conjointe étranger·e d'être naturalisé·e, autrement dit d'acquérir le statut de citoyen.ne possédant la pleine nationalité suisse. Toutefois, les mariages mixtes ne sont jamais considérés comme équivalents aux autres unions, et l'intégration des personnes naturalisées n'est que partielle et fragile. La déchéance de la nationalité suisse constitue une menace permanente : un·e Suisse naturaliséee dont le mariage prendrait fin pourra être soupçonné·e d'avoir fait un mariage blanc et verra sa naturalisation remise en question. Tout se passe comme si la situation marginale ne pouvait pas se résoudre, comme si le mariage restait, pour toujours, en dehors des 
normes conjugales. Dans le cas des familles lesboparentales et de la place qu'elles confèrent au donneur, I. Côté et al. montrent qu'à l'inverse, le temps peut modifier la situation originelle. Les décisions prises par les couples de femmes au sujet de la place du donneur au début du projet parental ne sont ainsi pas toujours respectées, transformant la façon dont les relations se nouent avec lui. Ces situations « hors normes » sont mouvantes et peuvent ainsi être transformées.

Enfin, dans la conclusion du numéro, Agnès Fine, anthropologue et historienne de la parenté, propose une lecture de ce dossier thématique à la lumière de ses propres travaux. Elle souligne combien il est heuristique de réinterroger les thématiques classiques de l'anthropologie, telles que l'alliance, le genre, les rapports entre vivant·e-s et défunt·e.s et la filiation, à l'aune de situations marginales. Ce numéro thématique, en déployant une réflexion pluridisciplinaire sur les marges dans l'étude de la parenté, a souhaité prolonger ce débat.

\section{Bibliographie}

CARSTEN J. (2000), « “Knowing where you've come from”: Ruptures and continuities of time and Kinship in narratives of adoption reunions », The Journal of the Royal Anthropological Institute, vol. 6, $\mathrm{n}^{\circ}$ 4, p. 687-703.

Carsten J. (2004), After kinship, Cambridge, Cambridge University Press.

Cherlin A. (1978), "Remarriage as an incomplete institution », American Journal of Sociology, 1978, vol. 84, $\mathrm{n}^{\circ}$ 3, p. 634-650.

Conklin B. A., Morgan L. M. (1996), « Babies, bodies, and the production of personhood in North America and a Native Amazonian society ", Ethos, vol. 24, n 4, p. 657-694.

CouRduriès J. (2016), « Ce que fabrique la gestation pour autrui. Les relations entre la femme porteuse, l'enfant et ses parents », Journal des anthropologues, vol. 1-2, $\mathrm{n}^{\circ} 144-$ 145, p. 53-76.

Douglas M. (1966), Purity and danger. An analysis of the concepts of pollution and taboo, Londres \& New York, Routledge.

Edwards J. (2006), " Reflecting on the "Euro" in "Euro-American" kinship: Lithuania and the United Kingdom », Acta Universitatis Klaipedensis, vol. 12, p. 129-139.

Edwards J., Franklin S., Hirsch E., Price F., Strathern M. (1999), Technologies of procreation: Kinship in the age of assisted conception, $2^{\mathrm{e}}$ éd., Londres \& New York, Routledge.

Fine A. (dir.) (1998), Adoptions : Ethnologie des parentés choisies, Paris, Éditions de la Maison des sciences de l'homme.

Fine A. (2002), « Qu'est-ce qu'un parent ? Pluriparentalités, genre et système de filiation dans les sociétés occidentales », Spirale, vol. 1, n²1, p. 19-43.

FRANKLIN S. (1997), Embodied progress: A cultural account of assisted conception, Londres \& New York, Routledge.

GENNEP A. van (1991 [1909]), Les rites de passage. Étude systématique des rites, Paris, Picard. 
GINSBURG F. D., RAPP R. R. (dir.) (1995), Conceiving the new world order: The global politics of reproduction, Berkeley, University of California Press.

Gluckman M. (1962), « Les rites de passage », in M. Gluckman (dir.) Essays on the Ritual of Social Relations, Manchester, Manchester University Press.

HéRAult L. (2011), « Le mari enceint : construction familiale et disposition corporelle », Critique, vol. 764-765, $\mathrm{n}^{\circ}$ 1, p. 48-60.

Kaufman S. R., Morgan L. M. (2005), « The anthropology of the beginnings and ends of life », Annual Review of Anthropology, vol. 34, p. 317-341.

LAVoIE K. (2019), Médiation procréative et maternités assistées: vers une approche relationnelle et pragmatique de la gestation pour autrui et du don d'ovules au Canada, thèse de doctorat, Montréal, Université de Montréal.

La Fontaine J. (1977), « The power of rites », Man, n 12, p. 421-437.

LÉvi-Strauss C. (1967 [1949]), Les structures élémentaires de la parenté, $2^{\mathrm{e}}$ éd., Paris, Mouton.

Malinowski B. (1963 [1922]), Les argonautes du Pacifique occidental, Paris, Gallimard.

Martial A. (2003), S’apparenter: Ethnologie des liens de familles recomposées, Paris, Éditions de la Maison des sciences de l'homme.

Martin A. (2019), « "Who do I look like?”: Kinning and resemblance in the experience of French donor conceived adults », Antropologia, 2019, vol. 6, n² 2 N.S., p. 45-62.

Mathieu S. (2017), « Donner et recevoir des embryons en France », Ethnologie française, 2017, vol. 167, n 3, p. 499-508.

Morgan L. H. (1985 [1877]), La société archaïque, 2e éd., Paris, Éditions Anthropos.

Porqueres i Gené E. (2001), « Le mariage qui dérange. Redéfinitions de l'identité nationale basque », Ethnologie française, vol. 31, n 3, p. 527-536.

SCHNEIDER D. (1984), A critique of the study of kinship, Ann Arbor, University of Michigan Press.

Strathern M. (1992), After nature. English kinship in the late twentieth century, Cambridge, Cambridge University Press.

THÉRY I. (2010), Des humains comme les autres. Bioéthique, anonymat et genre du don, Paris, Éditions de l'EHESS.

TuRner V. (1990 [1977]), Le Phénomène rituel. Structure et contre-structure, Paris, Presses Universitaires de France.

Wilgaux J. (dir.) (2005), « Qu'est-ce que la parenté ? Autour de l'œuvre de David M. Schneider $»$, Incidence, $\mathrm{n}^{\circ} 1$. 